\title{
Model Library of Polymer Electrolyte Membrane Fuel Cells for System Hardware and Control Design
}

\author{
Kevin L. Davies \\ Georgia Institute of Technology, Woodruff School of Mechanical Engineering \\ Atlanta, Georgia USA \\ Robert M. Moore Guido Bender \\ Hawaii Natural Energy Institute \\ Honolulu, Hawaii USA
}

\begin{abstract}
The trade-offs among dynamic response, efficiency, and robustness to external factors are fundamental to the optimization of hardware and controls for fuel cell systems. No previously published model of polymer electrolyte membrane fuel cells (PEMFCs) has the capability to simultaneously provide dynamic modeling capabilities, a clear representation of physical configurations, adjustable fidelity, and flexible interfaces. This paper presents the first such library, explains key aspects of the library's architecture, and demonstrates simulations under representative scenarios. The models, implemented in the acausal Modelica modeling language, are quasi-three-dimensional (quasi-3D), discretizing the fuel cell and its layers along the directions from the anode to the cathode and down the channel length. Keywords: fuel cell; system design; hardware; control
\end{abstract}

\section{Introduction}

Computer-based models are most useful in the design of hardware and controls for fuel cell (FC) systems if they provide the capability for dynamic simulation, clarity, adjustable fidelity, and acausal interfaces. Control design inherently concerns the dynamic response of a system, as the design of a FC system often requires a compromise between transient performance and efficiency. Thus the model's suitability is dependent on its ability to determine the system's dynamic response from the description of system components.

Hardware design often involves the consideration of multiple configurations. Because the configurations are typically chosen by humans, a model is most useful if it is represented in a clear, intuitive form. As illustrated in Figures 1a and $1 \mathrm{~b}$, the form of the model depends strongly on the modeling paradigm. The acausal schematic in Figure 1a directly corresponds to the physical connections between discrete components, therefore it is more understandable. Acausal models exist for all domains in which interactions among components are dominated by energy flow, including domains of mechanical, fluid, and thermal systems.

Typical design involves a top-down process of specification, from the system level to the component level, followed by a bottom-up process of validation (i.e., the system "Vee" [6]). Models used for all stages of design require adjustable fidelity, or the capacity for multiple levels of detail. During the process of specification at the system level, many configurations must be evaluated with limited resources, so that excessively detailed models are time-consuming, unfeasible, and unjustified. At the component level, however, the accurate representation of physical processes demands more detail.

Models should also have acausal interfaces so that they can be evaluated with different simulation inputs and outputs. The underlying equations for potentials and flows in physical systems are bidirectional, with no explicit specification of the order of calculations. The current through a $\mathrm{FC}$, for instance, is determined by bidirectional interactions between the FC and its electronic load (see Zenith et al. [14]). Most physical systems do not inherently have directionality and thus are best represented by acausal models. Acausal models allow inputs and outputs to be defined dur- 


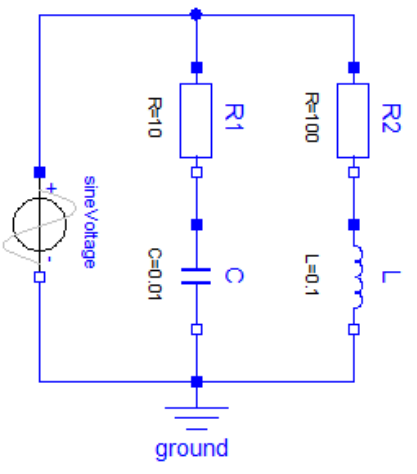

(a)

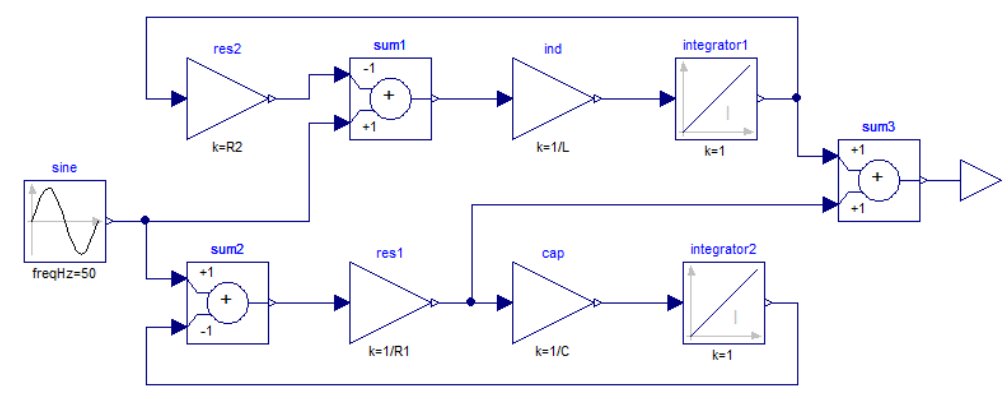

(b)

Figure 1: An electrical system represented as an (a) acausal diagram and a (b) causal diagram [7].

ing the final preparation for simulation, whereas causal and noncausal models have predefined inputs and outputs. Noncausal models have inputs and outputs that are opposite relative to a given causal model and should also be clearly distinguished from acausal models.

The Modelica language [8] supports acausal models. The models are expressed using differential algebraic equations (DAEs) and discrete events. Within the DAE formalism, the dynamic behavior of components is captured in terms of implicit ODE combined with algebraic constraints. The behavior of components is explicitly discretized over space, in contrast to a PDE formalism.

\section{Background}

In the early 1990s, Springer et al. [11], [10] and Bernardi et al. [1] established groundwork for modeling PEMFCs and related key model parameters to FC test results. The models continued to increase in sophistication, up to the inclusion of two-phase flow in three-dimensional (3D)s. In 2004, Weber and Newman [13] reviewed the rapidly growing body of literature on the transport mechanism within PEMFCs. The models range from the detailed description of specific processes to the coverage of the entire cell and take both first-principle and empirical approaches. However, as noted by Zenith et al. [14], many FC models consider the electronic current as an input. In fact, only four of the more than 250 published models [13] are known to be acausal. The model of Rubio et al. [9] provides an acausal and detailed representation of cell polarization. However, it lacks robustness, clear correlation to previous literature and the physical configuration of the
FC, fidelity with respect to the concentration gradient down the flow channel (it is one-dimensional (1D)) and consideration of the reactant streams as a mixed fluid, and only has electrical interfaces (no fluid or thermal interfaces). The model of Ungethüm [12] describes the entire FC system and has an acausal fluid network, but no details of the FC stack model have been published. The model described in this paper is a continuation of the work of Davies et al. [3, 4].

The approach and goal of the research described in this paper is to utilize state-of-the-art acausal modeling tools and methods to develop a robust model of a PEMFC that provides the capability for dynamic modeling, clarity, adjustable fidelity, and flexible interfaces.

\section{Model Components}

\section{$3.1 \quad$ FC Components}

The equations of the models in this section and subsequent sections are described in schematic form. At each physical interface node in the schematic (whether it is fluid, or thermal, or electrical), the flow variables sum to zero. This represents the balance equations for energy and chemical species. In the electrical case, for instance, this corresponds to Kirchhoff's Current Law. In addition, the across variables (e.g., electric potential, pressure, temperature) are set equal at every node. This implies that potential energy differences sum to zero around loops, which is analogous to Kirchhoff's Voltage Law. 


\subsubsection{Flow Plate}

Figure 2 provides a schematic for the FlowPlate, which consists of a network of PortVolume, PressureLoss, DiffusionSurface, ThermalConductor, ThermalConvection, HeatCapacitor, and HeatingResistor elemental models. The large interface icons along the boundary of the Figure represent the outside connections to the FlowPlate and the smaller icons in the interior represent the interfaces of the elemental models within the FlowPlate. The equations of the fluid network describe the pressure loss down the length of the channel, the storage of fluid in the channel volume, and the interface to the gas diffusion layer (GDL). The equations of the thermal network describe the heat conduction across the flow plate, the resistance heating and thermal energy storage in it, and the heat convection between the fluid and the flow plate. The equations of the electrical network describe the voltage loss across the flow plate, which may or may not be negligible, depending on the values of the parameters that represent the properties and thickness of the material.

The model captures pressure loss due to bulk flow along the reactant/product stream and down the channel (the $f$ direction). The contents of the dotted area are repeated for each of $N_{f}$ segments $\left(N_{f}=1\right.$ for a $1 \mathrm{D} \mathrm{FC}$ and $N_{f}>1$ for quasi-3D). Where the connection "wire" loops back on itself in Figure 2, the elements included in the loop are connected in series. The model assumes perfect electrical and thermal conduction in the $f$ direction, which is appropriate because the electronic flow and heat flow are predominately in the $z$ direction.

\subsubsection{Catalyst Layers}

Figure 3 is a schematic for the model of a cathode catalyst layer, CatLayer_ca, which contains of a network of ORR, MembraneSurface, Capacitor, ThermalConductor, HeatingResistor, HeatCapacitor, PoreVolume, TransportPorous, DiffusionMembrane, ElectroOsmoticDrag, and MembraneVolume elemental models. The physical meaning of the networks is similar to that of the FlowPlate. The contents of the outer dotted rectangle are duplicated $N_{f}$ times, and the contents of the inner dotted rectangles are duplicated $N_{z}+1$ and $N_{z}$ times. In this manner, the oxy- gen reduction reaction (ORR) is distributed over $N_{f}\left(N_{z}+1\right)$ instantiations of the ORR . The model assumes perfect fluid, thermal, and electrical insulation in the $f$ direction, which is appropriate because the transport within the flow plate dominates that of the other layers in the $f$ direction. The model of an anode catalyst layer, CatLayer_an, is identical except that it contains the HOR instead of the ORR and the media model represents hydrogen $\left(\mathrm{H}_{2}\right)$ and carbon monoxide $(\mathrm{CO})$ instead of oxygen $\left(\mathrm{O}_{2}\right)$ and nitrogen $\left(\mathrm{N}_{2}\right)$.

\subsubsection{GDL and PEM}

All of the elements of the GDL and PEM model are also contained within the CatLayer_ca. Like the CatLayer_ca, both of these models contain $N_{z}$ lumped segments through the thickness of the cell and $N_{f}$ segments in the direction along the length of the flow channel. The network of elemental models in the upper right of Figure 3 (ThermalConductor, HeatingResistor, HeatCapacitor, PoreVolume, and TransportPorous) represents the GDL . The thickness and porosity of each of the $N_{z}$ lumped segments through the GDL can be independently specified, making it possible to model a GDL with a microporous layer (MPL). Likewise, the network of elemental models in the lower right of Figure 3 (DiffusionMembrane, ElectroDsmoticDrag, and MembraneVolume), as well as ThermalConductor and HeatCapacitor, is representative of the PEM . The transport processes due to diffusion and electro-osmotic drag act in parallel. Electro-osmotic drag transports $\mathrm{H}_{2} \mathrm{O}$ from the anode to the cathode, in the direction of protonic flow. Both the electroosmotic drag process and generation of $\mathrm{H}_{2} \mathrm{O}$ from the ORR generally lead to a higher molar concentration of $\mathrm{H}_{2} \mathrm{O}$ on the cathode side, so there is a net diffusion of $\mathrm{H}_{2} \mathrm{O}$ from the cathode to the anode.

\section{$3.2 \quad$ FC}

Figure 4 represents the FC. It is a set of FlowPlate, GDL, CatLayer_an, CatLayer_ca, and PEM s connected through fluid, heat, and electrical interfaces. Each of these interconnections contains $N_{f}$ interfaces in parallel (one for each channel segment). There are alternative methods to describe numerous processes within the FC. These options allow the model's fidelity to be adjusted to suit 


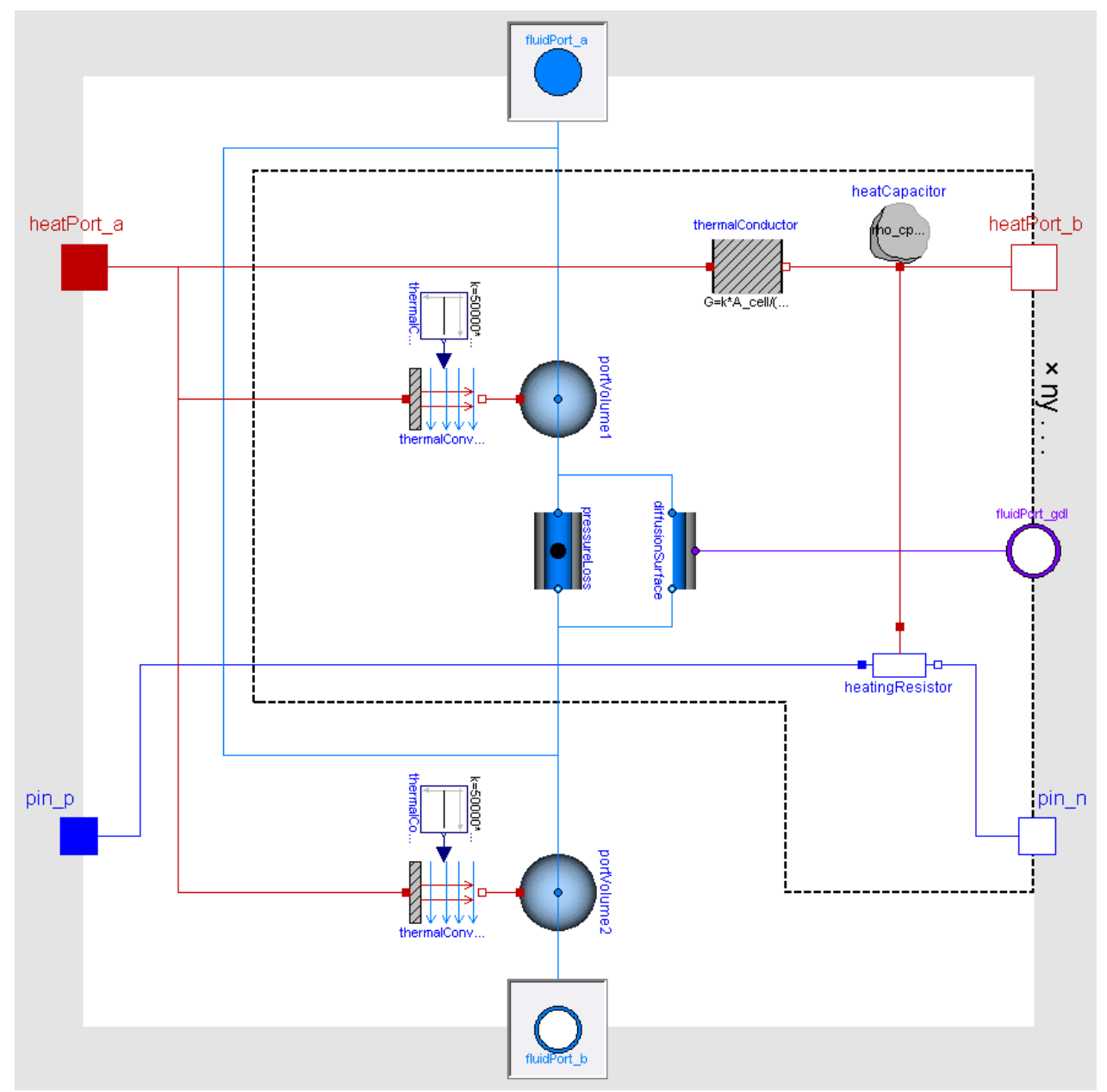

Figure 2: Schematic of the FlowPlate model.

the available simulation time and computational resources. Table 1 lists all of the options and notes the baseline options with asterisks.

\subsection{Test Scenarios}

Figure 5 shows the baseline test scenario used to evaluate the polarization curve of the model of a FC. The test scenario is essentially a model of an ideal FC test stand. The anode and cathode reactant streams are generated by the $\mathrm{Flu}-$ idSource_an and FluidSource_cas and are dissipated into the Boundary_an and Boundary_ca components, respectively. The $\mathrm{H}_{2} \mathrm{O}$ content of the reactant streams is specified in terms of relative humidity $(\mathrm{RH})$ or, alternatively, mole fraction of $\mathrm{H}_{2} \mathrm{O}$. The molar concentration of $\mathrm{O}_{2}$ in the dry cathode gas can be adjusted, allowing the FC to be simulated with air or $\mathrm{O}_{2}$. In the baseline scenario, the reactant flow rates are specified as constants in terms of equivalent current (i.e., the current corresponding to $100 \%$ utilization). Al- ternatively, the flow can be variable, specified in terms of an equivalence ratio and a current measurement. Concurrent or counter flow scenarios can be modeled by switching the cathode flow direction with the Reverser. The pressures at the anode and cathode outlets, as well as the temperatures at the exterior surface of the flow plates, are specified by constant signal sources. The FC is wired to an electrical circuit where the current is specified by a signal source and the electric potential is measured as the dependent variable. The signal source is a ramp function, and the ramp rate is small $\left(1 \mathrm{~mA} \mathrm{~cm}^{-2} \mathrm{~s}^{-1}\right)$ so that transient effects are negligible.

The test scenario is the highest level of the model. It provides the simulation stimuli as an input to the model under test (i.e., the FC). Therefore, the test scenario contains causal assignments, as shown by the arrows in Figure 4. In the baseline scenario, the signal sources are constants and ramp functions but could instead be data files, 


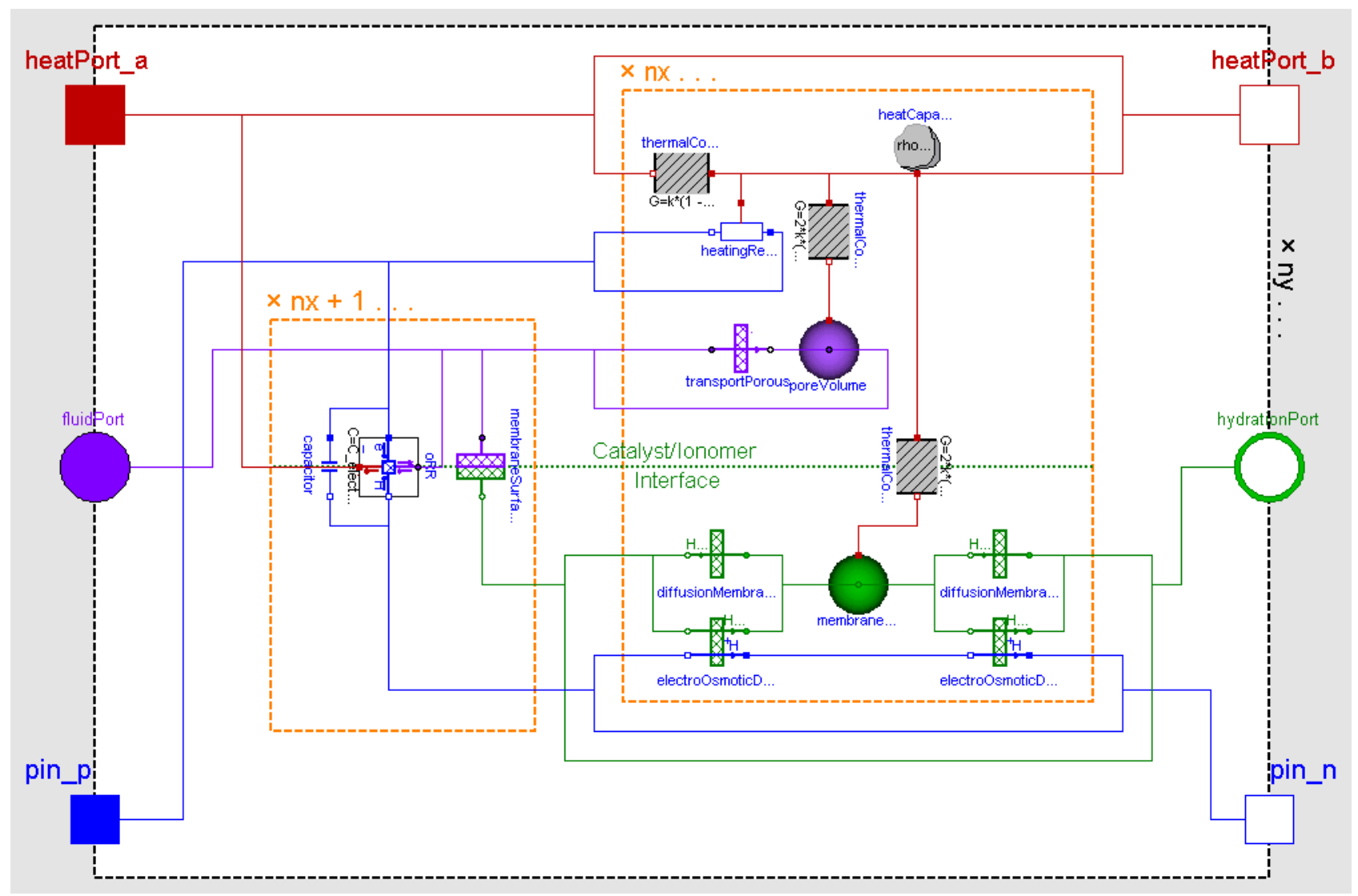

Figure 3: Schematic of the CaLayer_ca model.

inputs from a control system, or real-time signals from hardware-in-the-loop (HiL) equipment. Note that the acausal model formulation extends to the physical boundary of the system of interest, and causal stimuli are applied at the boundary. If the boundary was expanded to encompass a larger system, the causal stimuli would be applied at the new boundary (e.g., a vehicle drive profile applied to a FC vehicle model).

\section{Simulation Results}

The FC is evaluated by varying conditions and settings relative to a baseline scenario. Except where noted, the baseline conditions are used. Under the baseline scenario, the flow plates are held at $80^{\circ} \mathrm{C}$, and the anode|cathode reactant conditions are: $H_{2} \mid$ Air, $1.0|1.0 \mathrm{~atm}, 80| 80^{\circ} \mathrm{C}, 100 \mid 100 \% \mathrm{RH}$, and $2.0 \mid 3.0 \mathrm{~A} \mathrm{~cm}^{-2}$ equivalent current. The cell is $1 \mathrm{D}\left(N_{f}=1\right)$ and the baseline model options are noted by the asterisks in the third column of Table 1. Figure 6 shows the polarization of individual channel segments of a quasi-3D cell $\left(N_{f}=10\right)$ under anode|cathode reactant flows at constant flow rates of $1.4 \mid 1.4 \mathrm{~A} \mathrm{~cm}^{-2}$ equivalent current and at fixed equivalence ratios of $1.25 \mid 1.25$, with minimum equivalent current density of $0.18 \mid 0.18 \mathrm{~A} \mathrm{~cm}^{-2}$. Here, the fluid is assumed to be non-condensing (option 1.1a in Table 1), and the flow rates are low in order to exaggerate the difference in polarization between upstream and downstream segments. In both scenarios, as current density is increased, the upstream segments pass an increasingly disproportionate share of the current. This occurs due to a positive feedback mechanism within the cell, whereby increased current from the upstream segments leads to lower $\mathrm{O}_{2}$ molar concentration in the downstream segments, decreasing their performance and requiring increased current from the upstream segments in order to meet the current demand on the entire cell. As shown in Figure 6a, this mechanism can even cause the polarization of the downstream segments to fold back when the flow rate is fixed.

Figure 7a shows the effect of several model options from Table 1 . The polarization curves shown in the plot are the outliers among the set of all the polarization curves produced by varying only one option from the baseline. 
Table 1: Selectable model options

\begin{tabular}{|c|c|c|}
\hline Model Element & Characteristic & Option \\
\hline \multirow{4}{*}{ Media } & \multirow{2}{*}{$\mathrm{H}_{2} \mathrm{O}$ behavior } & 1.1a: Non-condensing \\
\hline & & 1.1b: *Condensing \\
\hline & \multirow{2}{*}{$\begin{array}{l}\text { Pressure-diffusivity } \\
\text { products }\end{array}$} & 1.2a: *Constant \\
\hline & & 1.2b: Dependent on temperature \\
\hline \multirow{6}{*}{ PressureLoss } & \multirow{4}{*}{ Flow regime } & 2.1a: *Laminar (valid for $R e<1000$ ) \\
\hline & & 2.1b: Quadratic turbulent (valid for $R e>4000$ ) \\
\hline & & $\begin{array}{l}\text { 2.1c: Laminar and quadratic turbulent (valid for } \\
R e<1000 \text { and } R e>4000 \text { ) }\end{array}$ \\
\hline & & 2.1d: Detailed (valid over full $R e$ range) \\
\hline & \multirow{2}{*}{$\begin{array}{l}\text { Fluid density and } \\
\text { dynamic viscosity }\end{array}$} & 2.2a: *Nominal \\
\hline & & 2.2b: Calculated from the media model \\
\hline \multirow{2}{*}{ TransportPorous } & \multirow{2}{*}{ Liquid $\mathrm{H}_{2} \mathrm{O}$ transport } & 3.1a: *Not included \\
\hline & & 3.1b: Included \\
\hline \multirow{2}{*}{$\mathrm{HOR}$} & \multirow{2}{*}{ Voltage } & 4.1a: *Constant \\
\hline & & $\begin{array}{l}\text { 4.1b: Thermodynamic (modified Butler-Volmer } \\
\text { equation) }\end{array}$ \\
\hline \multirow{3}{*}{ ORR } & \multirow{3}{*}{ Product $\mathrm{H}_{2} \mathrm{O}$} & 5.1a: Liquid \\
\hline & & 5.1b: Vapor \\
\hline & & 5.1c: *Two-phase \\
\hline \multirow{6}{*}{ PEM } & \multirow{3}{*}{ Protonic resistance } & 6.1a: Constant \\
\hline & & 6.1b: Dependent on current density \\
\hline & & 6.1c: *Dependent on hydration \\
\hline & \multirow{3}{*}{ Diffusion coefficient } & 6.2a: *Constant \\
\hline & & $\begin{array}{l}\text { 6.2b: Dependent on hydration, specified as a poly- } \\
\text { nomial }\end{array}$ \\
\hline & & $\begin{array}{l}\text { 6.2c: Dependent on hydration, specified as a lookup } \\
\text { table }\end{array}$ \\
\hline
\end{tabular}

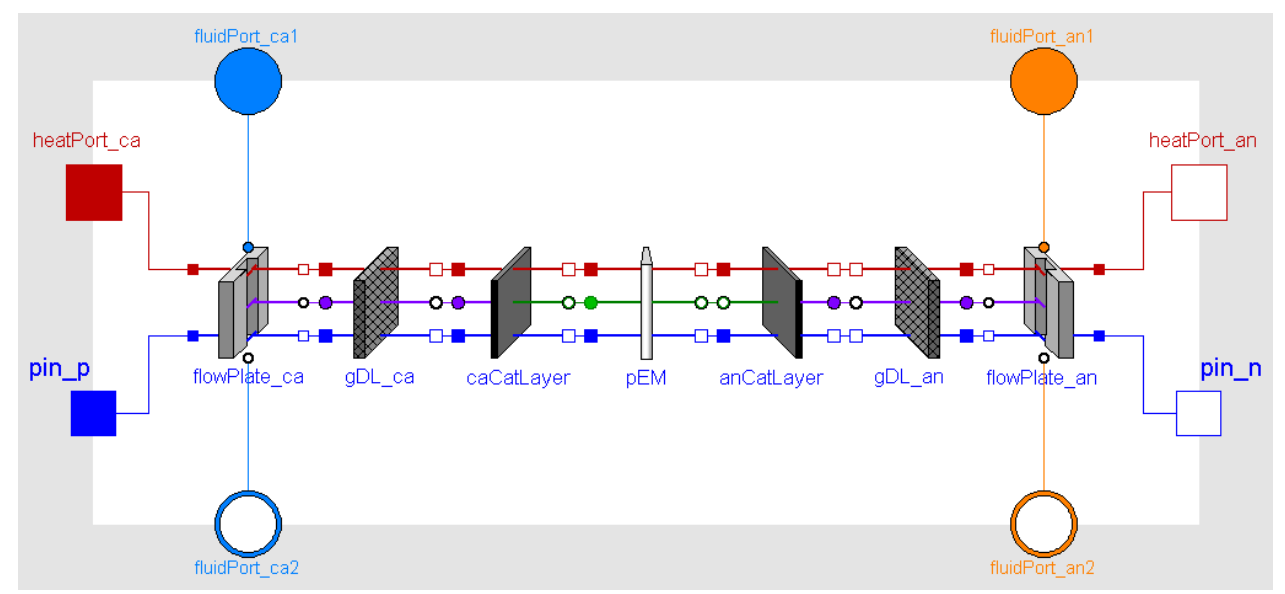

Figure 4: Schematic of the FC model. 


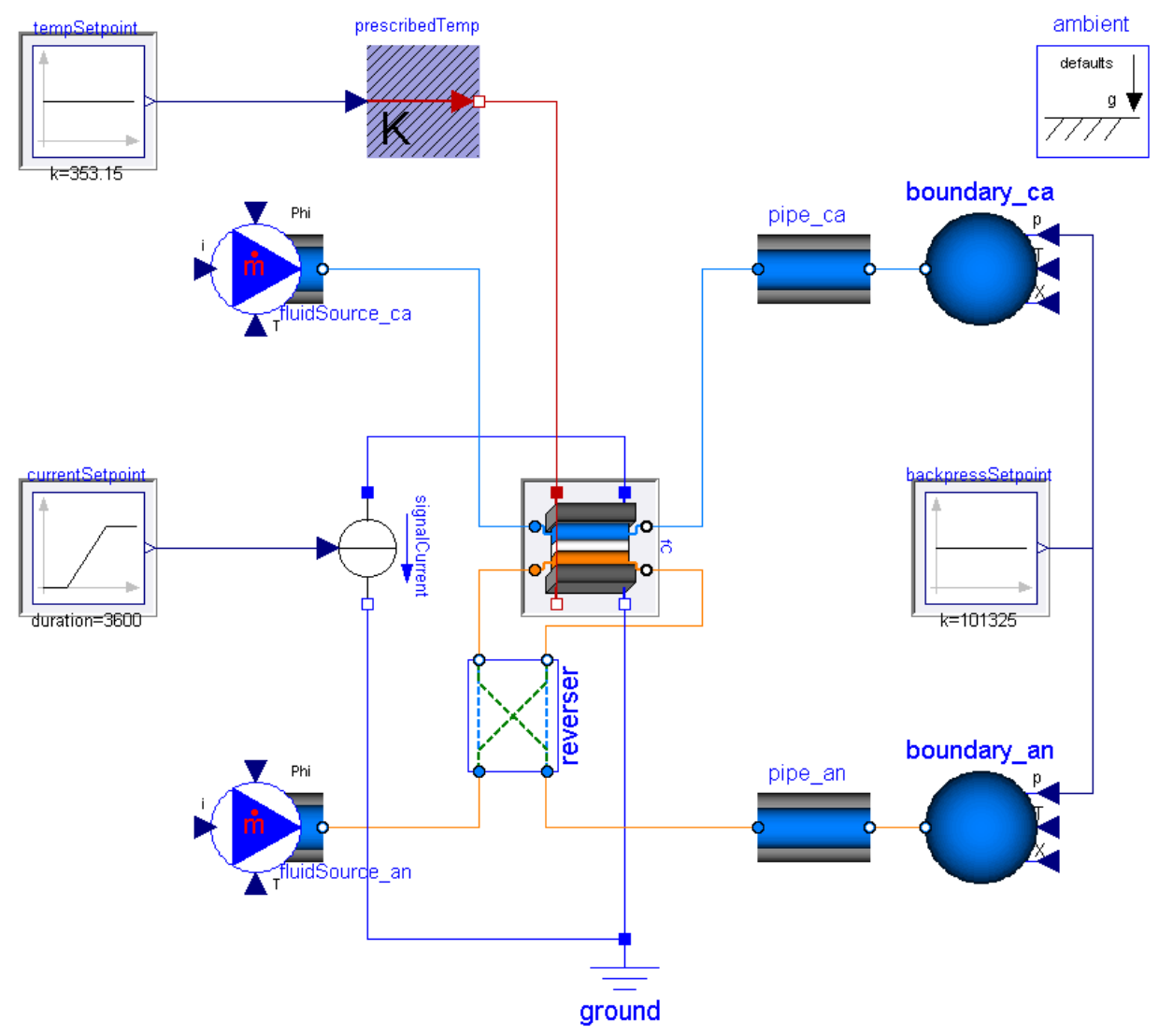

Figure 5: Schematic of the baseline test scenario.

The number of channel segments $\left(N_{f}\right)$ is another important model setting. As $N_{f}$ is increased, the model becomes more representative, but the simulation takes more time. Figure $7 \mathrm{a}$ shows the effect of $N_{f}$ on the polarization curve. As $N_{f}$ is increased, there is an apparent change in performance because the $\mathrm{O}_{2}$ molar concentration in each segment is calculated as the average of that at the inlet and outlet of the segment. The profile of $\mathrm{H}_{2} \mathrm{O}$ and $\mathrm{O}_{2}$ concentration is nonlinear down the channel length due to unequal current through the segments (as demonstrated in Figure 6). These concentrations affect the polarization via the overpotential in the cathode catalyst layer and the resistance loss in the polymer electrolyte membrane (PEM), and the effect is more pronounced where there is a large concentration gradient down the flow channel (e.g., at high currents). However, there is a limit beyond which there is negligible effect from increasing $N_{f}$, as shown in Figure 7a.

Figure 8 demonstrates the dynamics of the FC . The electronic load is cyclical, with a sinusoidal current from 0 to $1.5 \mathrm{~A} \mathrm{~cm}^{-2}$ with a $30 \mathrm{~s}$ period. Figure 8a shows the voltage losses of the cell. The cathode activation and concentration losses are dominant, and a small loop appears in the polarization curve due to the hysteresis in temperature at the cathode reaction site and the hydration of the PEM. Figure $8 \mathrm{~b}$ shows that the temperature vs. current hysteresis occurs counterclockwise. As current increases, the internal losses increase, increasing the temperature at the cathode catalyst layer. There is a lag in the temperature response, however, due to the heat capacities of the materials within the cell. The PEM hydration vs. current hysteresis occurs clockwise. As current increases, more $\mathrm{H}_{2} \mathrm{O}$ flows from the anode to the cathode due to electro-osmotic drag, resulting in a net drying effect of the PEM. There is a lag in the PEM hydration response since $\mathrm{H}_{2} \mathrm{O}$ is stored in the PEM material.

\section{Discussion}

The models simulate quickly enough to be manageable for design studies of the FC or combined with other models for studies of larger systems. However, any real-time application of the model, 


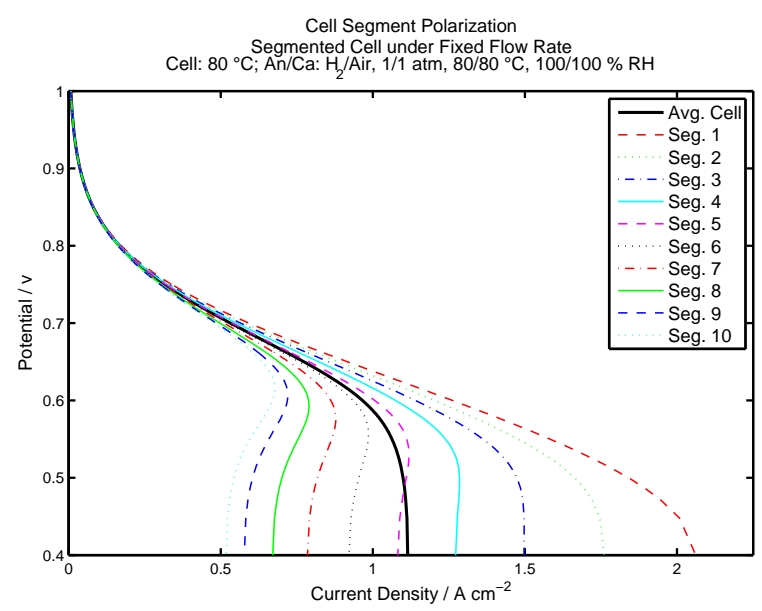

(a)

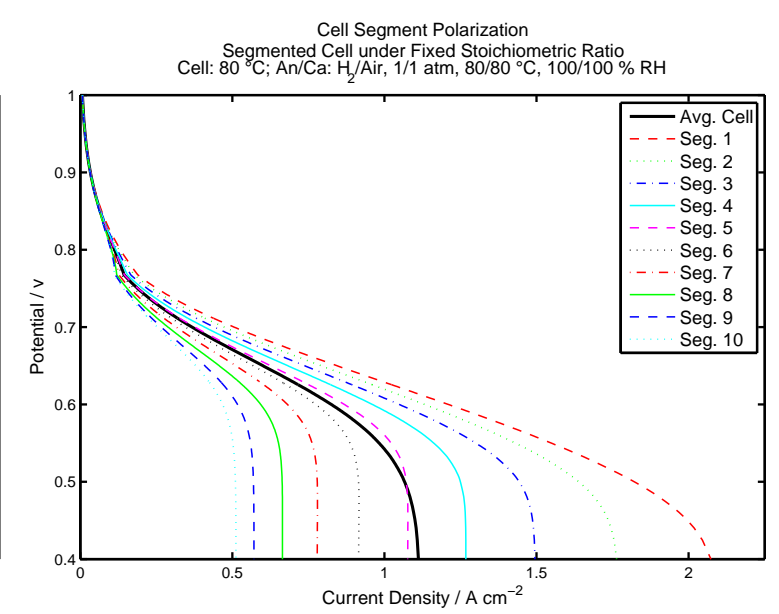

(b)

Figure 6: Cell segment polarization with anode/cathode reactants provided at (a) fixed flow rate of $2.0 \mid 2.0 \mathrm{~A} \mathrm{~cm}^{-2}$ and (b) fixed equivalence ratio of $1.25 \mid 1.25$.

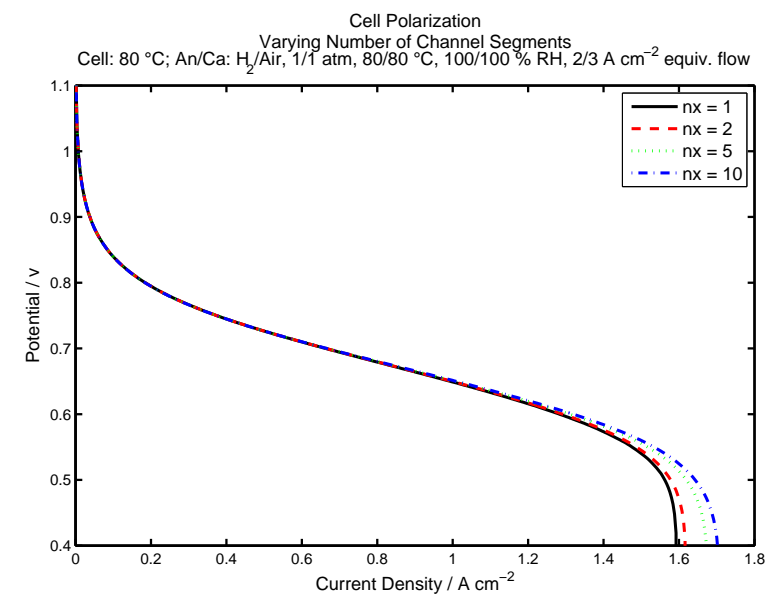

(a)

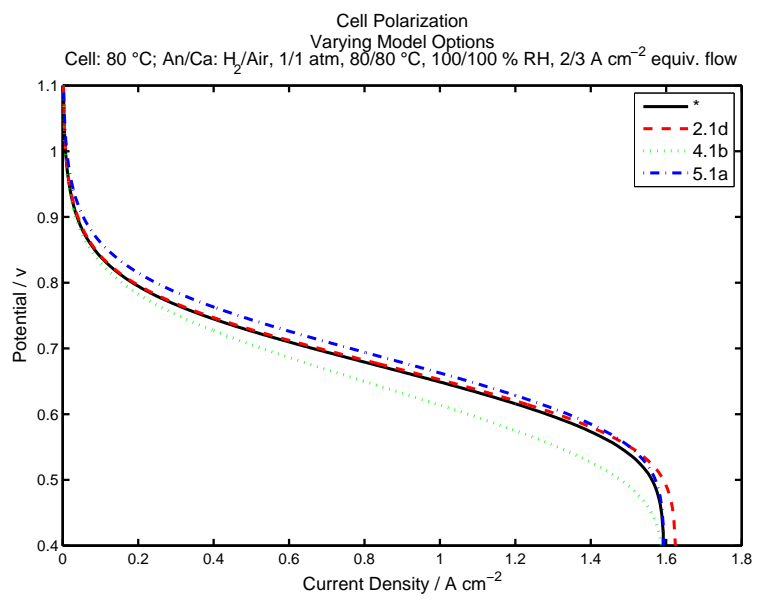

(b)

Figure 7: Effect of model options on the FC polarization curve: (a) selected model options from Table 1 and (b) number of channel segments.

e.g., for HiL or model based control (MBC), would likely require simplification or other adjustments. The baseline simulation has 31 time-varying state variables and 1595 nontrivial equations. Each additional channel segment in the quasi-3D case adds 23 states and 985 equations. As translated by the Dynamic Modeling Laboratory (Dymola) [5] and simulated with the Differential/Algebraic System Solver Library (DASSL), the baseline simulation requires $1.6 \mathrm{~s}$ to run on an Intel Centrino Duo T7300 2.0 GHz based computer.

The model translator performs symbolic manipulation on the model so that the simulation is manageable. Several guidelines have been used in the design of the model library so that this simpli- fication is possible. First, storage elements (e.g., capacitors or media storage volumes) are placed at nodes where processes are connected to reduce the size of nonlinear systems of implicit DAEs. This introduces state variables, and the model translator is then able to break the equations into smaller sets which can be calculated independently over each simulation time step. Second, media storage volumes are generally not connected directly, but rather through processes, to avoid index reduction steps in the model translation [2]. Third, the models provide provisions to manage model stiffness (where the time constants of the model vary widely) via the model options listed in Table 1. Fourth, the model equations are carefully 


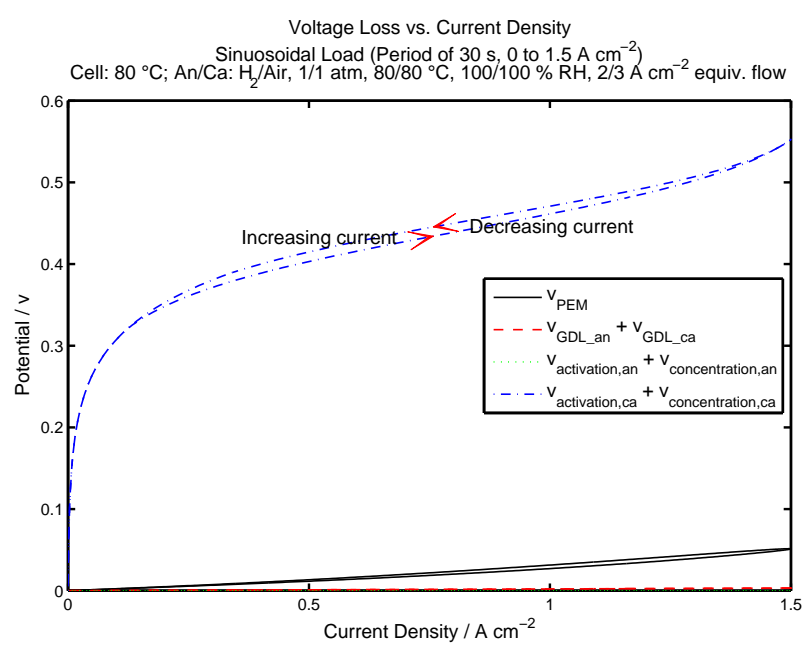

(a)

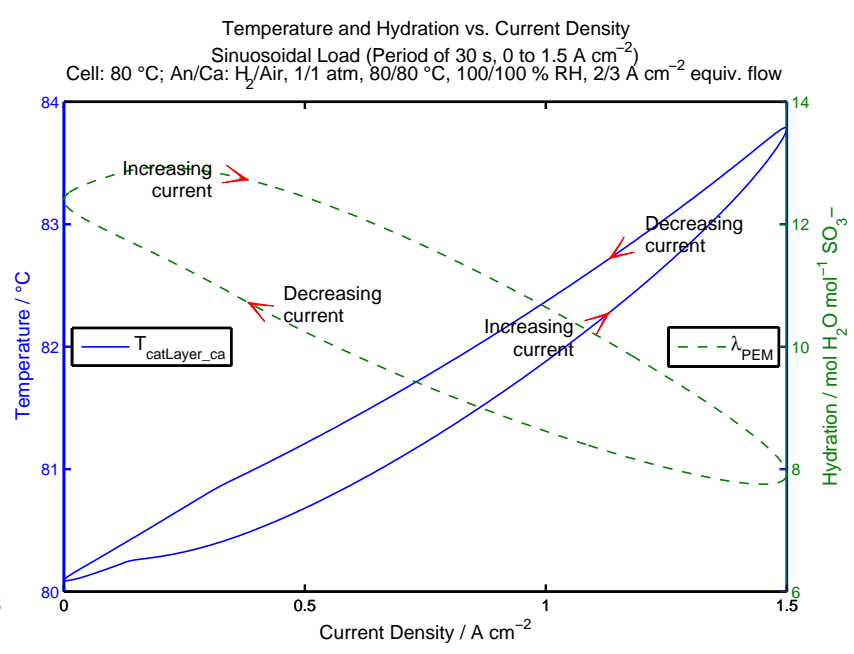

(b)

Figure 8: FC response with a sinusoidal load: (a) losses due to resistance, activation, and concentration in the PEM, GDL, and catalyst layers and (b) cathode catalyst layer temperature and PEM hydration.

formulated to reduce the sensitivity to numerical precision, as in the TransportPorous, for example.

The model avoids or carefully handles discrete events where the structure of the model equations changes abruptly, as in the two-phase behavior of $\mathrm{H}_{2} \mathrm{O}$. This gives the model translator more flexibility in the symbolic manipulation of the model equations, and it avoids the need for the simulator to reinitialize when the events occur. When a discrete event occurs, the simulation solver must reinitialize, and by default it begins a guess process with the values of the state variables before the event occurred. However, the discrete events that occur when switching between one-phase and two-phase regions still slow down the simulation. When the quasi-3D model of a FC is used with $N_{f}=10$, events occur frequently enough to increase the simulation time significantly. For that reason, $\mathrm{H}_{2} \mathrm{O}$ condensation is not included unless absolutely necessary.

\section{Conclusion}

As described in the introduction, computer-based models are most useful for the hardware and control design of FCs if they provide the capability for dynamic simulation, clarity, adjustable fidelity, and acausal interfaces.

The model library presented here addresses these requirements by modeling the $\mathrm{FC}$ as a system of acausal, object-oriented models specified in terms of DAEs in the Modelica language. This paper provides a high-level view of how the models are structured and presents results from several usage scenarios. The research demonstrates the power of combining the empirical and theoretical knowledge in the field of FCs with a state-of-theart tool for acausal modeling.

In the hardware design of a FC system, there is often a trade-off between attributes of the FC (e.g., efficiency or performance metric) and the balance of plant (e.g., parasitic loss or economic cost). The results demonstrate the ability of the model to evaluate the efficiency of the FC, even with variable causality.

\section{Acknowledgments}

This research was supported in its initial stages by the Office of Naval Research under grant \#N00014-04-0682 to the Hawaii Natural Energy Institute and later by the Robert G. Shackelford Fellowship of the Georgia Tech Research Institute. The authors wish to acknowledge the support of the Modelica Association and the Modelica community by the open sharing of the Modelica Standard Library and Modelica_Fluid. Although it is not currently possible to offer the library described within this paper online as open source, the authors request that any interested readers contact them. 


\section{References}

[1] D. M. Bernardi and M. W. Verbrugge. A mathematical model of the solid-polymerelectrolyte fuel cell. Journal of The Electrochemical Society, 139(9):2477-91, 1992.

[2] F. E. Cellier and E. Kofman. Continuous System Simulation. Springer, New York, NY, 2006.

[3] K. Davies and R. Moore. Object-oriented fuel cell model library. ECS Transactions, 11(1):797, 2007.

[4] K. Davies and R. Moore. PEMFCSim: A fuel cell model library in Modelica. In Fuel Cell Seminar, Austin, TX, 2007.

[5] Dynasim AB. Dymola: Dynamic modeling laboratory, 2007. v6.2.

[6] K. Forsberg and H. Mooz. System engineering for faster, cheaper, better. The Center for Systems Management, Reprinted by SF Bay Area Chapter of INCOSE, http://www.incose.org/sfbac/, 1998.

[7] Modelica Association. Modelica: A Unified Object-Oriented Language for Physical Systems Modeling: Tutorial. Linköping, Sweden, ver. 1.4 edition, December 2000.

[8] Modelica Association. Modelica: A unified object-oriented language for physical systems modeling, February 22005.

[9] M. A. Rubio, A. Urquia, L. GonzÃ $\tilde{j}_{j} l e z$, D. Guinea, and S. Dormido. FuelCellLib: A modelica library for modeling of fuel cells. In 4th International Modelica Conference, Hamburg-Harburg, Germany, March 2005. Modelica Association.

[10] T. E. Springer, M. S. Wilson, and S. Gottesfeld. Modeling and experimental diagnostics in polymer electrolyte fuel cells. Journal of The Electrochemical Society, 140(12):35133526, 1993.

[11] T. E. Springer, T. A. Zawodzinski, and S. Gottesfeld. Polymer electrolyte fuel cell model. Journal of The Electrochemical Society, 138(8):2334-2342, 1991.
[12] J. Ungethüm. Fuel cell system modeling for real-time simulation. In 4 th International Modelica Conference, HamburgHarburg, Germany, March 2005. Modelica Association.

[13] A. Z. Weber and J. Newman. Transport in polymer-electrolyte membranes III: Model validation in a simple fuel-cell model. Journal of The Electrochemical Society, 151(2):326339, 2004.

[14] F. Zenith, F. Seland, O. E. Kongstein, B. Borresen, R. Tunold, and S. Skogestad. Controloriented modelling and experimental study of the transient response of a high-temperature polymer fuel cell. Journal of Power Sources, 162(1):215-227, 2006. 